\title{
Automated Diffraction Tomography: A New Era for Electron Crystallography
}

\author{
A. A. Stewart,* E. Mugnaioli, T. E. Gorelik, I. Andrusenko, and U. Kolb \\ * Institute for Physical Chemistry, Johannes Gutenberg University Mainz, Jakob Welder Weg 11, \\ Mainz, Germany. stewarta@uni-mainz.de
}

The advent of Automated Diffraction Tomography (ADT) as a method for collecting electron diffraction data sets has heralded a paradigm shift in the ability of electron crystallography to collect and solve structures from a wide range of materials [1], including, inorganic, organic, mineralogy, and hybrid materials. Not only has it lead to the structure solution of over 30 structures in 2 years but has been achieved without the need for imaging or any other additional information, only the standard small molecule approach of using direct methods for structure solution.

The ADT approach has several advantages over selected area and nanobeam techniques including minimizing dose to the specimen, something which is most critical when dealing with beam sensitive compounds particularly organics, select individual crystals from an agglomerated sample, especially important when dealing with specimens from inorganic synthesis. These advantages are achieved by conducting the experiments in STEM mode, both acquisition of the diffraction patterns and low resolution imaging of the crystal for tracking during a tilt series with minimum hysteresis. Utilizing all these advantages it has been possible to obtain and solve structures from crystals as small as $20 \mathrm{~nm}$, which opens the door to electron crystallography becoming a powerful tool in nanotechnology research.

While the traditional approach for electron crystallography has been to collect zonal diffraction patterns with limited reciprocal space coverage so as the corresponding images can be used to supplement structure solution with phases we have shown that mimicking the X-ray experiments and collecting as full as reciprocal space coverage as possible has a 2 fold benefit. 1. It increases the number of recorded reflections which can be as high as $100 \%$ depending on the space group. 2 . The off axis data has a reduced dynamical content compared to the zone axis data, even with precession.

The ADT experiments come in two flavors, one which takes only slices through reciprocal space and the second which uses precession to sweep through reciprocal space and fully integrate the reflections. The latter has a higher success rate of structure solution but the former has also been shown to be useful when no precession unit is available. It is important to note here that the use of precession is not to take the beam off axis because the crystal is already off axis by experimental design, but only to help fully integrate the recorded reflections, for a more complete analogy with oscillating crystal experiments in X-ray crystallography.

We will present theory and practice of how Automated Diffraction Tomography experiments are currently implemented, along with numerous examples of structure solutions from electron diffraction data sets. As well as discuss areas for further improvement of this young and exciting field of electron crystallography. 


\section{References}

[1] E. Mugnaioli, et al., Ultramicroscopy. 109 (2009) 758.

[2] C. Birkel et al., Journal of the American Chemical Society. 132 (2010) 9881.

[3] We thank the SFB 625 granted by the DFG for financial support, and to Max Otten (FEI) for help in programing the acquisition module. 